\title{
Description et cycle biologique de Tetrameres (Tetrameres) cardinalis n. sp.
}

\author{
par J. C. QUENTIN et N. BARRE \\ Laboratoire de Zoologie (Vers) associé au C.N.R.S., \\ Muséum national d'Histoire naturelle, 43, rue Cuvier, F 75231 Paris Cedex 05 \\ et Laboratoire central de recherches, 22, rue Pierre-Curie, 94700 Maisons-Alfort
}

\section{Résumé.}

Description d'une nouvelle espèce de Nématode Spiruride Tetrameres (Tetrameres): cardinalis n. sp. parasite du Cardinal de Virginie Richmondia cardinalis. Ce Tetrameres présente: des structures céphaliques primitives; 33 à 43 paires d'épines somatiques chez le mâle; des spicules dont la longueur est de $365-440 \mu$ et de 65 à $350 \mu$; des œufs dont la coque est pourvue de drux touffes de filaments.

Ce Spiruride évolue expérimentalement chez le Criquet Locusta migratoria. Son développement larvaire est caractérisé par la rapidité de l'organogenèse, la stabilité des structures. céphaliques du troisième stade à l'adulte, la petite taille $(800 \mu)$ des larves infestantes, la. localisation des larves dans le tissu adipeux de l'Insecte.

\section{Summary.}

\section{Description and Life History of Tetrameres (Tetrameres) cardinalis sp. n.}

A new species of Spirurid Nematode Tetrameres (Tetrameres) cardinalis n. sp. parasiteof a Cardinal: Richmondia cardinalis is described. This Tetrameres shows primitive cephalic structures, 33 to 43 pairs of somatic spines in male, spicules of unequal length: left $365-440$, right $65-350 \mu$, mature eggs with tufts of polar filaments.

The life-cycle of this Spirurid has been obtained experimentally with the grass-hopper Annales de Parasitologie humaine et comparée (Paris), t. $51, \mathrm{n}^{\circ} 1$ 
Locusta migratoria as an intermediate host. Its larval development is characterized by the quickness of organogenesis, the stability of cephalic structures from third stage to adult stage, the small size $(800 \mu)$ of infective larva, the localization of larva in the insect fat tissue.

\section{Introduction}

L'adaptation à la vie tissulaire entraîne chez les Nématodes du genre Tetrameres un profond dimorphisme sexuel dans l'aspect général du corps et chez de nombreuses espèces dans les structures céphaliques des mâles et des femelles.

Sur le plan de la biologie, le déroulement du cycle de ces Spirurides chez l'hôte définitif semble comparable entre les différentes espèces: le parasite migre et se développe dans la paroi du proventricule des Oiseaux. Chez l'hôte intermédiaire par contre, le comportement et la localisation des larves peut différer selon l'espèce étudiée.

Ainsi les larves infestantes de Tetrameres crami Swales, 1933 et de T. fissispina (Diesing, 1851) se développent dans le tissu musculaire de Copépodes (Swales, 1936 ; Garkavi, 1949), celles de T. americana Gram, 1927 migrent et s'encapsulent dans les muscles de différents Orthoptères (Gram, 1931) alors que les larves infestantes de T. mohtedai Bhalerao et Rao, 1944 évoluent dans le tissu adipeux d'Orthoptères (Sundaram et coll., 1963) ou dans la membrane entourant les viscères abdominaux de Lépidoptères (Lim, 1975).

Chaque espèce de Tetrameridae peut donc être caractérisée à la fois par sa morphologie et par le comportement de ses larves chez l'hôte intermédiaire.

Nous étudions dans ce travail la morphologie et la biologie d'un Tetrameres dont $13 q$ et $20 \sigma^{\star}$ ont été récoltés dans la paroi du proventricule d'un Cardinal de Virginie, originaire du Mexique.

\section{Description}

Les structures apicales du mâle (fig. $1 \mathrm{~B}, \mathrm{C}$ ), présentent un cycle céphalique de quatre papilles globuleuses, deux amphides, un cycle labial de quatre papilles masquées en partie dans le cadre de la capsule buccale, un cycle labial interne à la base de six dents chitinoïdes situées à l'apex de la capsule buccale ; celle-ci en vue apicale, a un contour hexagonal et semble formée sur sa hauteur de trois éléments (fig. $1 \mathrm{D}$, E).

Chez la femelle (fig. 2), les structures céphaliques sont plus réduites. On observe (fig. 2 C) quatre papilles céphaliques, deux amphides et un cycle labial interne situé sur le bourrelet de la capsule buccale. Celle-ci paraît s'être étirée en profondeur du fait du rétrécissement de la bouche (fig. $1 \mathrm{D}$ ), mais on retrouve les trois éléments remarqués chez le mâle. 


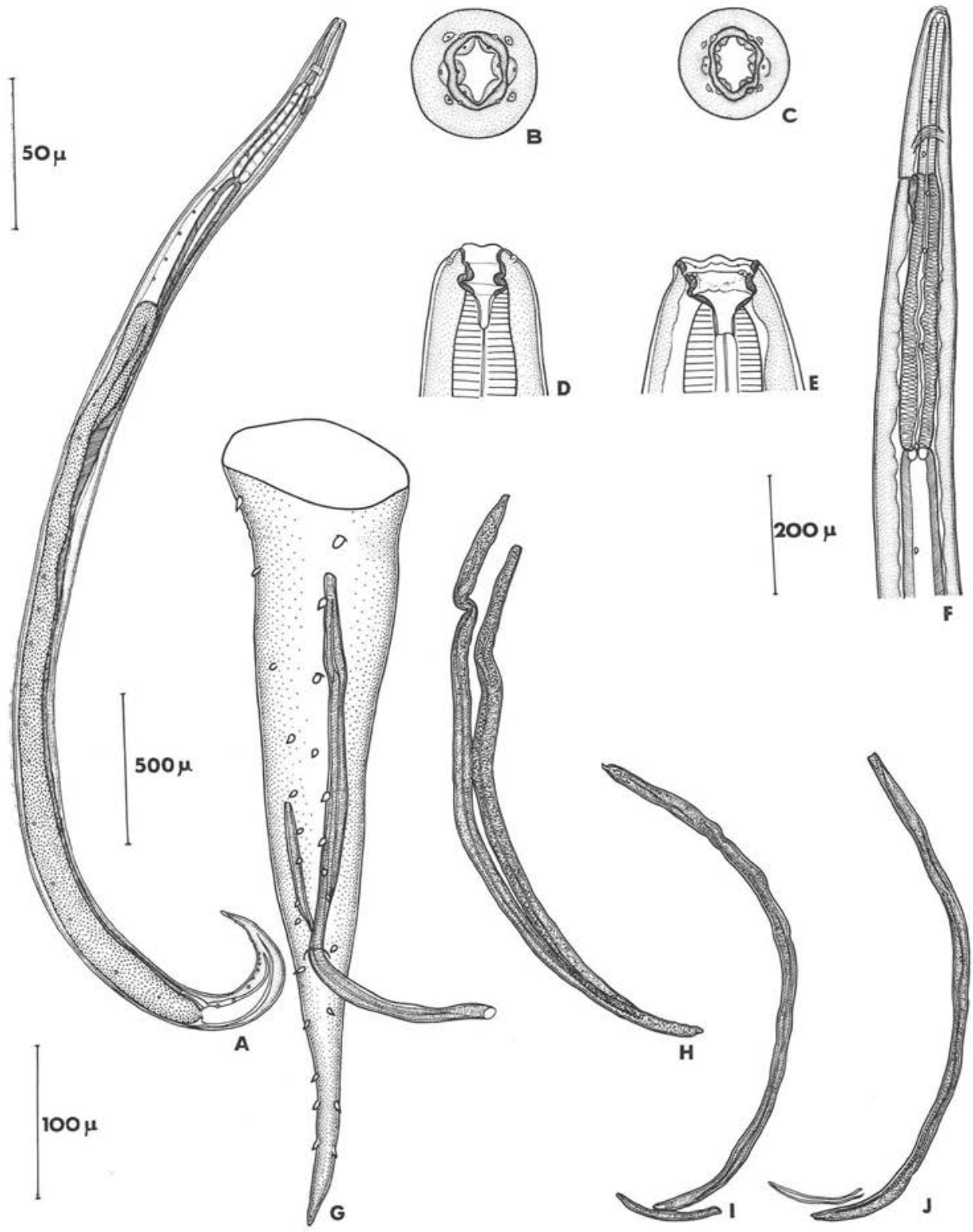

FIG. 1. - Tetrameres (Tetrameres) cardinalis n. sp. రో. A : mâle représenté en vue latérale; $\mathrm{B}$ et $\mathrm{C}$ : deux têtes représentées en vue apicale; $\mathrm{D}$ : tête, vue dorsale; $\mathrm{E}$ : tête, vue latérale; F : extrémité antérieure du corps, vue latérale; G : bourse caudale du mâle représentée en vue ventrale; H, I, J : spicules de différents mâles.
A : éch. $500 \mu ;$ B, C, D, E : éch. $50 \mu ;$ F : éch. $200 \mu ;$ G, H, I, J : éch. $100 \mu$. 


\section{Mâles.}

Le corps grêle et incolore porte en arrière des deirides et sur toute la longueur du corps deux rangées de fines épines régulièrement espacées dont le nombre varie de 33 à 43 paires selon les spécimens. Le chiffre le plus couramment observé est de 39 paires. Quatre à cinq paires d'épines sont post-cloacales. La longueur des mâles atteint 4,2 à $4,95 \mathrm{~mm}$.

\section{Mâle holotype.}

Ses dimensions sont les suivantes: longueur $4,4 \mathrm{~mm}$; largeur $140 \mu$; anneau nerveux, deirides et pore excréteur situés respectivement à $175 \mu, 220 \mu$ et $270 \mu$ de l'apex ; capsule buccale profonde de $12 \mu$; longueur de l'œsophage musculaire $225 \mu$; longueur de l'œsophage glandulaire $400 \mu$; distance du coude testiculaire à l'apex $1,14 \mathrm{~mm}$; longueur du spicule droit $80 \mu$, longueur du spicule gauche $380 \mu$ (longueur des spicules relevés sur d'autres spécimens : 65 et $365 \mu$., $72 \mu$ et $385 \mu$., $85 \mu$ et $400 \mu, 350 \mu$ et $440 \mu$ ); longueur de la queue $170 \mu$.

\section{Femelles.}

Les femelles sont globuleuses, d'un rouge foncé et présentent une striation cuticulaire dilatée (fig. $2 \mathrm{~A}, 2 \mathrm{~B}$ ). La région œsophagienne et l'extrémité caudale dépassent du corps. Le corps est gonflé par l'intestin qui est lui-même entouré par les deux ovaires et les branches utérines. L'ovéjecteur s'ouvre en avant de l'anus. Au cours de l'embryogenèse, la structure coquillière de l'œuf se modifie. On voit apparaître à chacun des pôles deux zones de plus faible épaisseur (fig. $2 \mathrm{H}$ ). Celles-ci vont constituer deux opercules (fig. $2 \mathrm{I}$ ) sur lesquels seront fixées deux touffes de filaments (fig. $2 \mathrm{~J}$ ). Les femelles ont des dimensions comparables.

\section{Femelle allotype.}

Ses dimensions sont les suivantes: longueur $4,6 \mathrm{~mm}$; largeur $3,4 \mathrm{~mm}$; longueur de l'extrémité antérieure saillante $380 \mu$; anneau nerveux, et pore excréteur situés respectivement à 150 et $200 \mu$ de l'apex; profondeur de la capsule buccale $12 \mu$; longueur de l'œsophage musculaire $110 \mu$, longueur de l'œsophage glandulaire $180 \mu$; vagin situé à $540 \mu$ de la pointe caudale, longueur de la queue $340 \mu$.

\section{Discussion}

Ces Tetrameres se classent dans le sous-genre Tetrameres (Creplin, 1846) en raison de l'absence d'appendices cuticulaires latéraux dans la région cervicale du corps. et de la présence de deux rangées latérales d'épines chez le mâle. 
Par ailleurs, la morphologie de ces spécimens se définit chez le mâle par le nombre de ces épines, la longueur des spicules, l'absence de différenciations labiales, et chez la femelle par l'aspect des œufs munis à chacun des pôles d'une touffe de filaments.
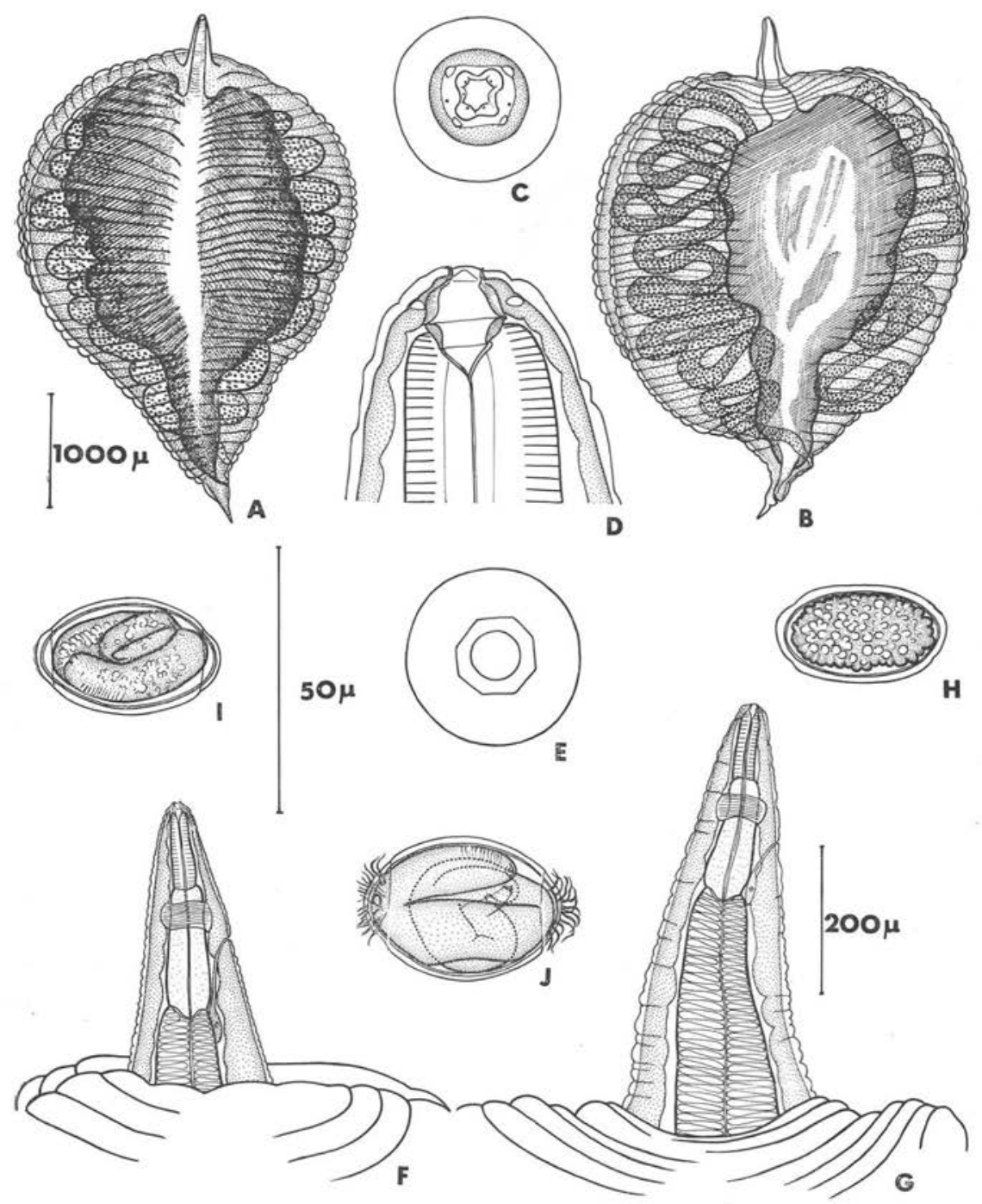

FIG. 2. - Tetrameres (Tetrameres) cardinalis n. sp. ㅇ. A, B : femelles; C: tête représentée en vue apicale; D: tête représentée en vue latérale; E: tête, coupe optique au niveau de la capsule buccale; $F$ et $G$ : extrémité antérieure du corps de deux femelles; $\mathrm{H}$, I, et $\mathrm{J}$ : œufs représentés à différents stades de l'embryogénèse.

A, B : éch. $1000 \mu$; C, D, E, H, I, J : éch. $50 \mu$; F, G : éch. $200 \mu$. 
Ces éléments permettent de rapprocher nos spécimens de trois espèces :

- Tetrameres dubia Travassos, 1917, parasite de Gallinago paraguaiae et Capella gallinago en Russie (Oshmarin, 1956). Cette espèce se différencie de notre matériel par la petite taille des mâles $(1,6 \mathrm{~mm})$ et par la longueur deux fois plus grande du spicule gauche $(720 \mu)$.

En outre chez cette espèce, les épines ne se trouvent que dans la région antérieure du corps alors qu'elles sont disposées sur toute la longueur du mâle sur nos spécimens.

- $T$. coloradensis Schmidt, 1962 présente chez le mâle quatre rangées possédant chacune 33 à 34 paires d'épines somatiques. Il n'existe que deux rangées sur nos spécimens. Les spicules gauche et droit mesurent $777 \mu$ et $67 \mu$ alors que ces dimensions sont respectivement de $365-400 \mu$ et de $65-85 \mu$ sur nos spécimens mâles qui sont deux fois plus longs que ceux de Schmidt.

- T. paucispina Sanground, 1928 a été récolté chez un Oiseau d'Amérique du Sud Amblyramphus holosericeus. Le plus long spicule mesure 328 à $371 \mu$, le plus petit 12 à $154 \mu$.

Sandground n'a observé des épines somatiques qu'au niveau des $2 / 3$ postérieurs du corps, où elles sont au nombre de 25 au plus, dont 3 sont post-cloacales.

Harwood, 1933 identifie à T. paucispina des Tetrameres parasites de Richmondia cardinalis au Texas. Il remarque toutefois deux rangées d'épines cuticulaires qui, sur certaines de ses préparations, sont visibles dans la région œsophagienne du mâle, mais ne précise pas la longueur des spicules ni les dimensions de ses spécimens.

Nos spécimens diffèrent des types de $T$. paucispina décrits par Sandground, par la répartition des épines somatiques sur le corps du mâle, et par le plus grand nombre de ces épines dans les régions pré et post-cloacales.

Par ailleurs, la taille des femelles est plus petite chez $T$. paucispina $(2,38 \mathrm{~mm})$ que sur nos spécimens $(4,6 \mathrm{~mm})$.

Nous pensons donc que les spécimens que nous avons récolté chez Richmondia cardinalis appartiennent à une espèce distincte de $T$. paucispina et que cette espèce est nouvelle. Nous proposons de la nommer T. cardinalis n. sp.

Les spécimens observés par Harwood, $1933 \mathrm{chez} R$. cardinalis au Texas qui diffèrent des types de $T$. paucispina par la répartition des épines somatiques, pourraient appartenir également à $T$. cardinalis.

\section{Cycle biologique}

\section{Matériel et méthodes.}

1. CROISSANCE du PARASITE CHEZ L'Hôte INTERMÉdiaIRe.

Des femelles de $T$. cardinalis sont dilacérées et des fragments d'utérus sont placés entre les pièces buccales de criquets: Locusta migratoria L. 

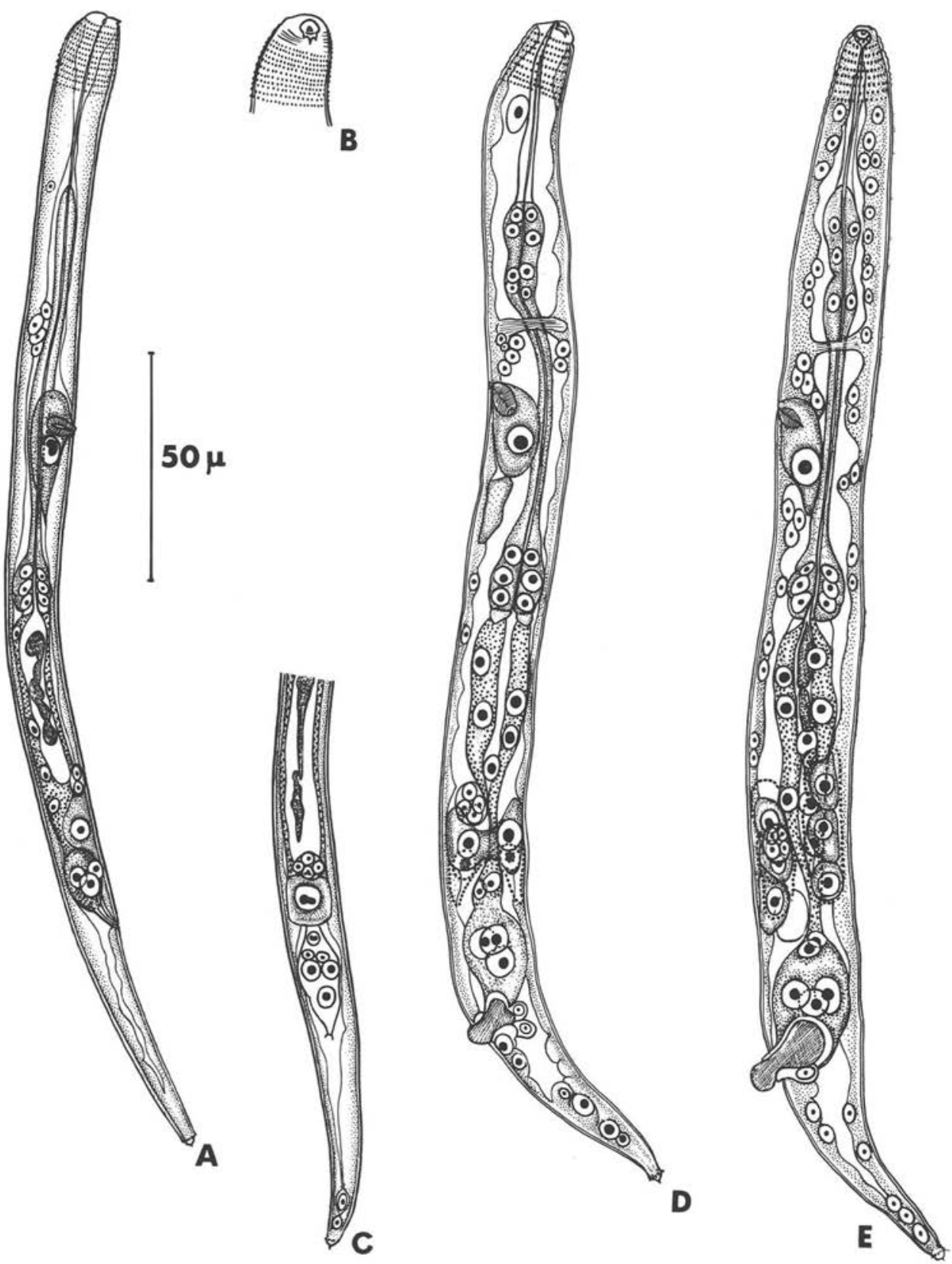

FIG. 3. - Tetrameres (Tetrameres) cardinalis n. sp. (premier stade larvaire). A : larve après $24 \mathrm{~h}$ de développement; B : détail de l'extrémité apicale et du crochet céphalique sir la face latérale gauche; C: extrémité postérieure de la larve, vue ventrale ; D : larve âgée de $48 \mathrm{~h}$; on note l'apparition dans la région caudale de la larve de quatre cellules issues de $\mathrm{R} 1$; $\mathrm{E}$ : larve âgée de $48 \mathrm{~h}$ à un degré plus avancé de l'organogénèse (accroissement des noyaux intestinaux).
A, B, C, D, E : éch. $50 \mu$. 
2. CRoissance du parasite CHEZ L'Hôte définitiF.

Un Cardinal de Virginie infesté expérimentalement en deux fois à trois jours d'intervalle avec successivement 14 larves et 30 larves infestantes est disséqué 11 jours après la deuxième infestation. De nombreuses larves du quatrième stade mâles et femelles résultant de l'infestation de 11 jours ainsi qu'une jeune femelle que nous pensons âgée de 14 jours sont récoltées à la surface du proventricule.

\section{Allure du développement.}

- Chronologie des mues.

A la température de $28^{\circ} \mathrm{C}$ le cycle de ce Spiruride évolue rapidement chez l'Insecte. $24 \mathrm{~h}$ après l'infestation, des larves sont récoltées dans la cavité générale. A quatre jours, l'exuvie du premier stade larvaire est décollée dans les régions céphaliques et caudales (fig. $4 \mathrm{~B}$ ) et, au cinquième jour du développement, les larves sont au deuxième stade larvaire. La deuxième mue débute vers le septième jour. L'exuvie est largement décollée dans la région postérieure de la larve (fig. $5 \mathrm{C}$ ) et laisse apparaître l'ornementation caudale du troisième stade. Quelques-unes de ces larves sont alors situées dans le tissu adipeux. Onze jours après l'infestation, de nombreuses larves infestantes sont observées dans le tissu adipeux, dans de minces capsules réactionnelles mesurant environ $400 \times 200 \mu$.

\section{- Croissance larvaire.}

Les larves mesurent au début du développement $250 \mu$ de long sur $13 \mu$ de large et atteignent $350 \mu$ de long sur $28 \mu$ de large au début du deuxième stade larvaire. Les larves infestantes ont une longueur de $800 \mu$ sur $38 \mu$ de large.

\section{Morphologie et organogénèse des stades larvaires.}

L'organogenèse de ce Nématode est particulièrement rapide. On observe en effet la division de la cellule $\mathrm{R} 1$ et l'augmentation du nombre des cellules intestinales dès le deuxième jour.

\section{PREMIER STADE.}

A $24 \mathrm{~h}$, la larve présente dans la région antérieure douze à quinze rangées d'épines cuticulaires et un trident chitinoïde situé sur le bord gauche de l'ouverture buccale (fig. 3 B). L'extrémité caudale est entourée d'une fine couronne d'épines.

L'anatomie de cette larve (fig. $3 A, C$ ) se résume en une ébauche œsophagienne qui occupe plus de la moitié de la longueur du corps, une volumineuse cellule excrétrice, une ébauche intestinale où l'on perçoit difficilement quatre ou cinq noyaux 

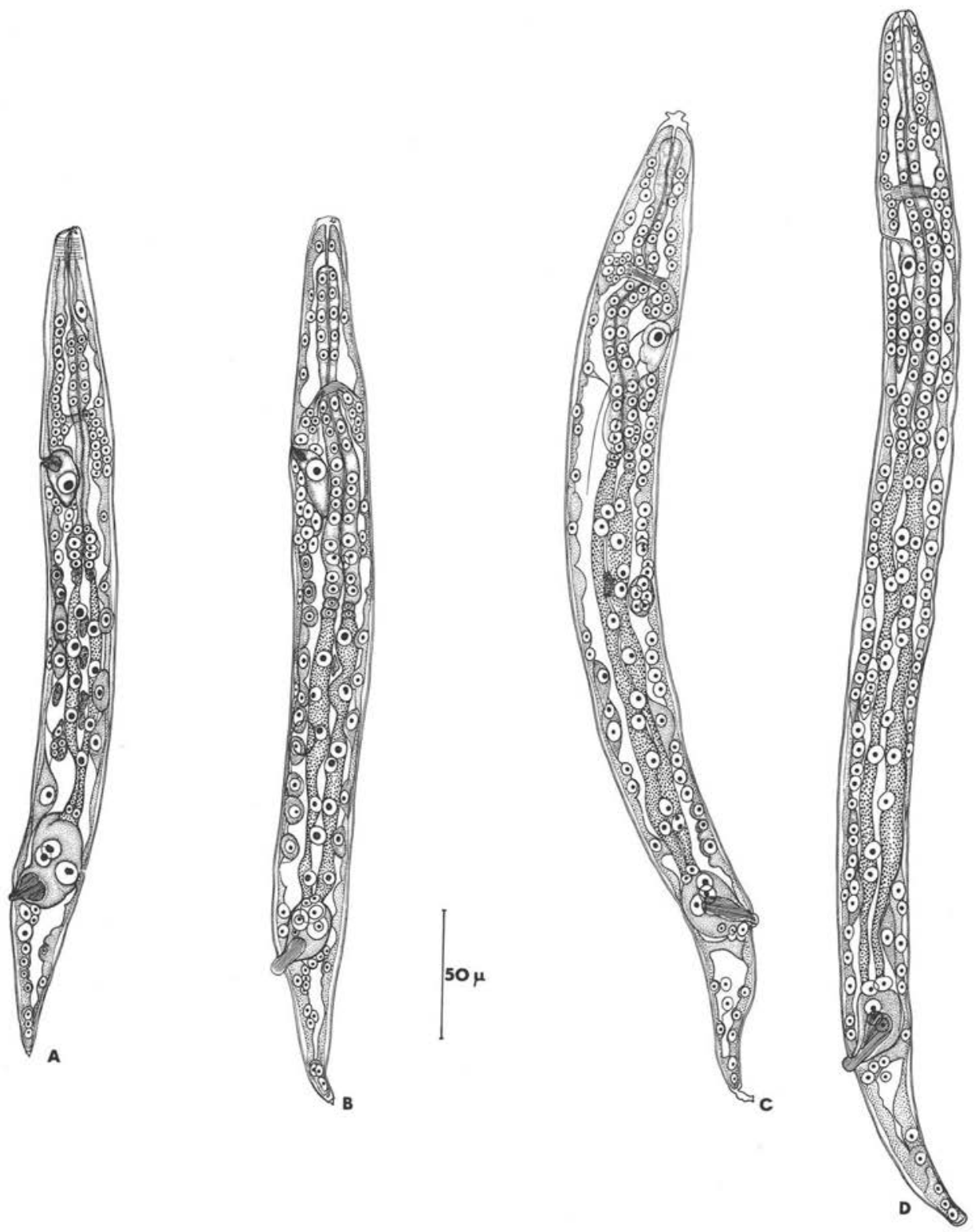

Fig. 4. - Tetrameres (Tetrameres) cardinalis n. sp. (fin du premier stade larvaire, début du deuxième stade). A: larve du premier stade âgée de 3 jours: B: larve, fin du premier stade âgée de 4 jours; on note le décollement de l'exuvie dans les régions céphaliques et caudales; $\mathrm{C}$ : larve au début du deuxième stade après 5 jours de développement, enfermée dans l'exuvie du premier stade; D: larve au deuxième stade, débarrassée de son exuvie ( 5 jours de développement).

A, B, C, D : éch. $50 \mu$. 
intestinaux, une volumineuse cellule $\mathrm{R} 1$ et trois cellules rectales $\mathrm{R} 2, \mathrm{R} 3, \mathrm{R} 4$, regroupées en avant du pore anal.

Nous identifions les quatre petites cellules situées en avant de la cellule $\mathrm{R} 1$ à l'ébauche génitale.

Les dimensions de cette larve sont les suivantes: longueur $257 \mu$; largeur $13 \mu$; pore excréteur situé à $90 \mu$ de l'apex; longueur de l'ébauche œsophagienne $136 \mu$; longueur de l'ébauche intestinale $50 \mu$; longueur de l'ébauche rectale $23 \mu$; cellule $R / 1$ située à $24 \mu$ du pore anal; longueur de la queue $50 \mu$.

Après $36 \mathrm{~h}$ de développement (fig. $3 \mathrm{D}$ ), chacune de ces ébauches s'épaissit. La cellule R 1 s'est divisée et a donné naissance à quatre cellules entourant la partie postérieure de l'intestin. Les cellules rectales accroissent leur volume et secrètent un bouchon rectal. Cette larve mesure $272 \mu$ de long sur $18 \mu$ de large.

Chez la larve âgée de 48 h (fig. 3 E), l'ébauche intestinale compte huit noyaux. Les cellules issues de R 1 se sont multipliées et s'incorporent à la paroi du corps.

Peu de modifications anatomiques surviennent chez la larve âgée de 3 jours, si ce n'est l'accroissement du volume des cellules rectales.

Cette larve mesure $325 \mu$ de long sur $28 \mu$ de large.

\section{DeuxiÈme STADE.}

Au quatrième jour (fig. $4 \mathrm{~B}$ ), la première mue s'accompagne d'une transformation de l'ébauche œsophagienne, qui perd sa forme rhabditoïde et s'accroît vers la région céphalique, et un allongement de l'intestin, qui compte environ douze cellules intestinales.

Les dimensions de cette larve sont: longueur $350 \mu$; largeur $29 \mu$; anneau nerveux et pore excréteur situés à $65 \mu$ et $88 \mu$ de l'apex; longueur de l'œsophage $165 \mu$; longueur de l'intestin $115 \mu$; ébauche génitale située à $90 \mu$ en avant de l'anus; longueur du rectum $30 \mu$; longueur de la queue $60 \mu$.

La larve âgée de 5 jours (fig. 4 C) est un deuxième stade larvaire, long de $390 \mu$, large de $38 \mu$, qui compte 17 cellules intestinales.

La différenciation de l'œsophage en une région glandulaire plus étroite en avant de l'anneau nerveux et une région glandulaire plus épaisse, s'observe sur la larve âgée de 6 jours (fig. 4 D). Cette larve mesure $484 \mu$ de long et $30 \mu$ de large.

Au septième jour du développement (fig. 5 B), l'organisation interne de la larve est presque celle d'une larve de troisième stade. L'œsophage musculaire s'est allongé et occupe toute la partie située en avant de l'anneau nerveux; l'ébauche génitale est plus antérieure, les épines caudales du troisième stade apparaissent sous la cuticule.

Les dimensions de cette larve, qui appartient encore au deuxième stade par sa capsule buccale très petite, sont les suivantes: longueur $810 \mu$; largeur: $35 \mu$; anneau nerveux et pore excréteur situés respectivement à 100 et $125 \mu$ de l'apex; longueur de 

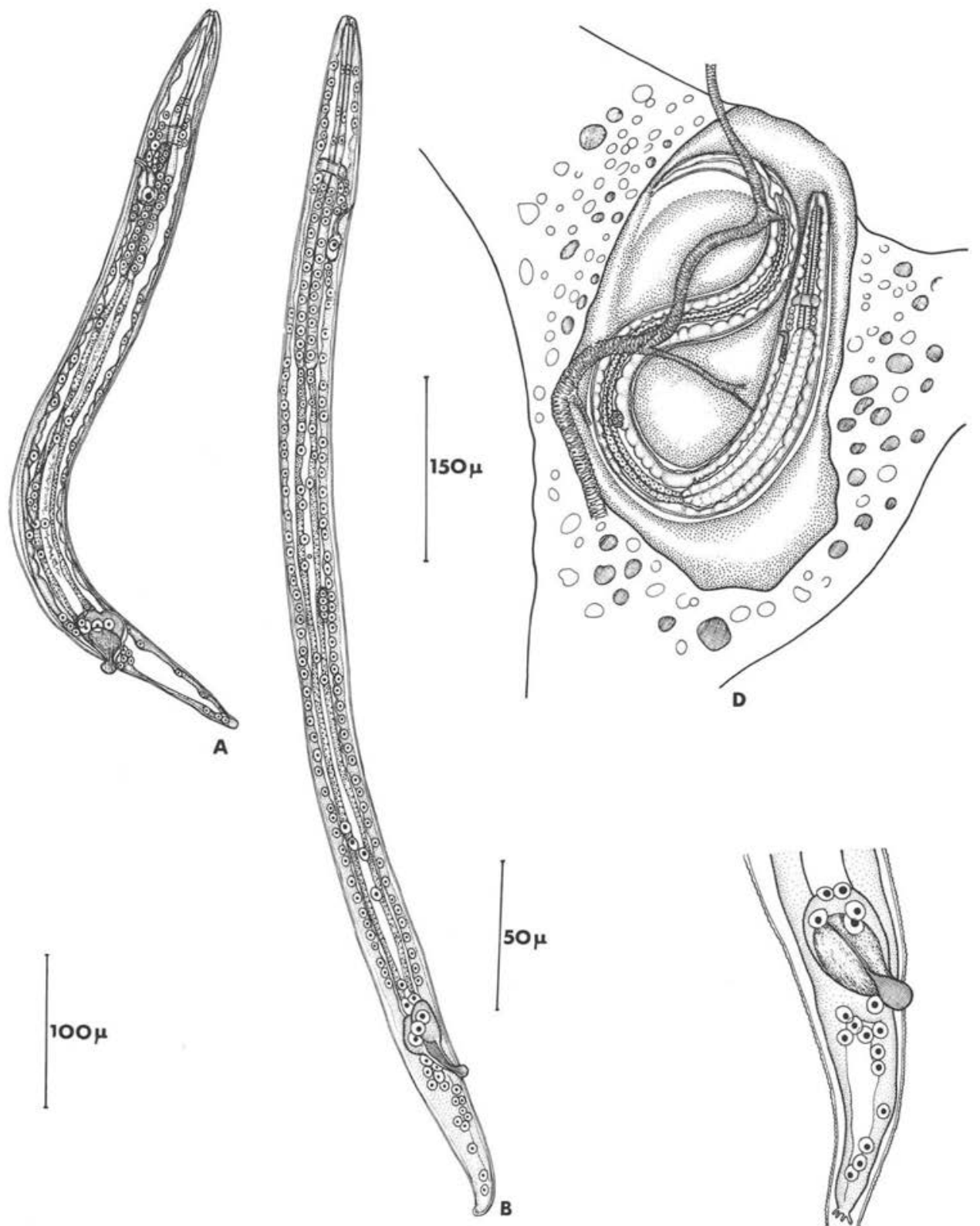

A

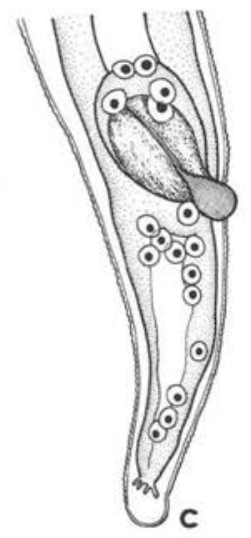

FIG. 5. - Tetrameres (Tetrameres) cardinalis n. sp. (deuxième stade larvaire). A: larve du deuxième stade âgée de 6 jours; B : larve âgée de 7 jours (fin du deuxième stade); on remarque l'accroissement de l'intestin et le décollement de l'exuvie dans la région caudale ; $\mathrm{C}$ : détail de la région caudale d'une larve en train de muer; D : larve encapsulée dans un lobe du tissu adipeux; on note le faible développement de la capsule.

$$
\text { A, B : éch. } 100 \mu \text {; C : éch. } 50 \mu \text {; D : éch. } 150 \mu \text {. }
$$


l'œsophage musculaire: $100 \mu$; longueur de l'œsophage glandulaire: $137 \mu$; longueur de l'intestin $425 \mu$; ébauche génitale située à $315 \mu$ en avant de l'anus; longueur du rectum $50 \mu$; longueur de la queue $100 \mu$.

\section{TROISIÈME STADE.}

La particularité de la larve du troisième stade (fig. O) réside à la fois dans ses structures céphaliques, cuticulaires et caudales.

- Structures céphaliques (fig. $6 \mathrm{~B}, \mathrm{C}$ ). La capsule buccale est aplatie latéralement et le cadre buccal rectangulaire porte six minuscules terminaisons du cycle labial interne. Il est entouré par quatre papilles labiales externes, par quatre papilles céphaliques et deux amphides.

- Structures cuticulaires (fig. 6 D). Les stries cuticulaires sont profondément marquées et donnent à la cuticule un aspect crénelé.

- Extrémité caudale (fig. $6 E, F, G$ ). Elle est ornée de 5 à 8 pointes cuticulaires.

Sur le plan de l'organogénèse, une coloration au bleu de Toluidine montre que l'œsophage glandulaire est constitué d'une partie interne riche en cellules et d'un manchon cytoplasmique où les cellules sont moins nombreuses. L'intestin est très long et compte environ 80 à 100 cellules.

Les dimensions de cette larve sont: longueur $800 \mu$; largeur $38 \mu$; anneau nerveux, deirides et pore excréteur situés respectivement à $90 \mu, 110 \mu$ et $115 \mu$ de l'apex ; profondeur de la capsule buccale $10 \mu$; longueur de l'œsophage musculaire $105 \mu$; longueur de l'œsophage glandulaire $177 \mu$; ébauche génitale située à $320 \mu$ de l'anus; longueur de l'intestin $380 \mu$; longueur du rectum $38 \mu$; longueur de la queue $103 \mu$.

\section{QUATRIÈME STADE.}

Les larves obtenues après un développement de 11 jours chez le Cardinal appartiennent à des quatrièmes stades mâles et femelles (fig. $7 \mathrm{~A}, \mathrm{~B}$ ). Ces larves se différencient des larves infestantes par leur capsule buccale agrandie, dont le rebord atteint le cycle labial externe, et par leur région caudale, qui ne présente aucune ornementation.

Elles diffèrent entre elles par la position de leur ébauche génitale. L'ébauche du testicule se développe au niveau de la région antérieure de l'intestin (fig. $7 \mathrm{~L}$ ) et celle de l'ovéjecteur dans la région postérieure (fig. $7 \mathrm{~N}$ ).

Les dimensions de ces larves, comparables dans les deux sexes, sont les suivantes: longueur 870-880 $\mu$; largeur $30 \mu$; anneau nerveux, deirides et pore excréteur situés respectivement à $90-100 \mu, 110-120 \mu, 110$ à $130 \mu$ de l'apex ; capsule buccale profonde de $10 \mu$; longueur de l'œsophage musculaire 115-130 $\mu$; longueur de l'œsophage glandulaire $170-190 \mu$; ébauche testiculaire longue de $70 \mu$, distante de l'anus de $310 \mu$; ébauche génitale femelle longue de $123 \mu$, vulve distante de l'anus de $80 \mu$; longueur de la queue $80-100$ u. 

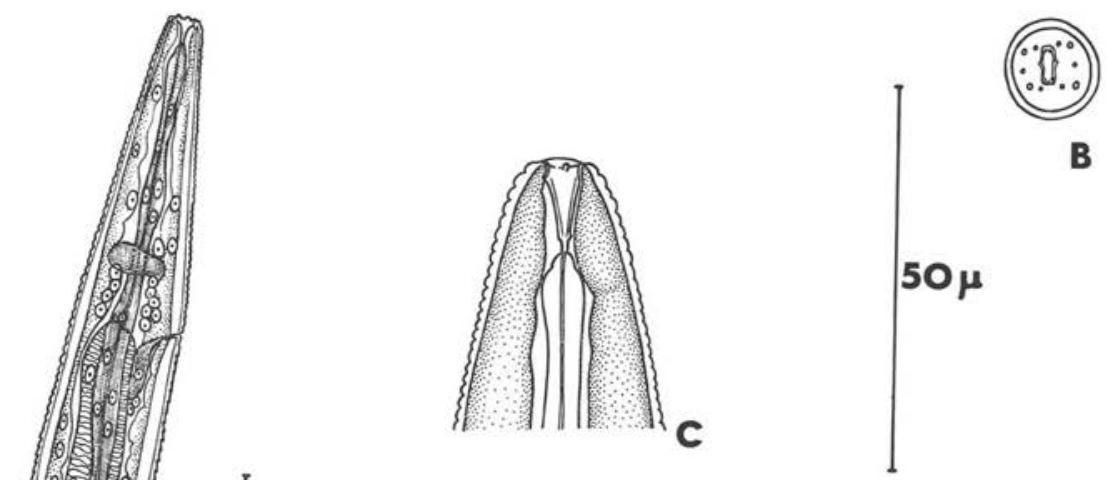

$100 \mu$

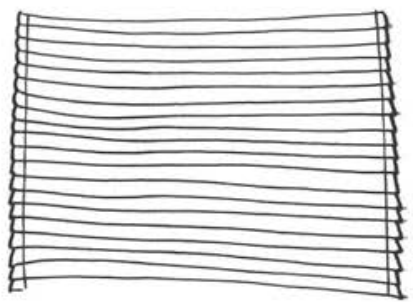

D
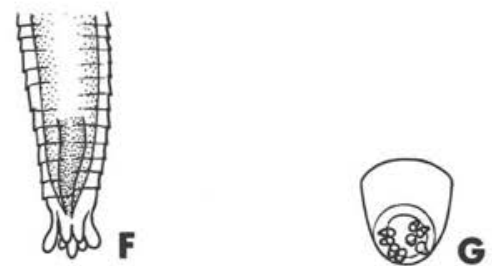

A

FIG. 6. - Tetrameres (Tetrameres) cardinalis n. sp. (troisième stade larvaire). A : larve du troisième stade (11 jours de développement); les noyaux sont mis en évidence par une coloration au bleu de Toluidine; on remarque les deux parties de l'œsophage glandulaire et le grand nombre de noyaux intestinaux; B : tête de larve infestante en vue apicale; le rebord de la capsule buccale est allongé dorso-ventralement et porte six minuscules papilles labiales internes; C : détail de la capsule buccale en vue latérale; D : détail de la striation cuticulaire ; E, F, G : détail de l'ornementation caudale en vue latérale, ventrale et apicale. A : éch. $100 \mu ; \mathrm{B}, \mathrm{C}, \mathrm{D}, \mathrm{E}, \mathrm{F}, \mathrm{G}$ : éch. $50 \mu$. 

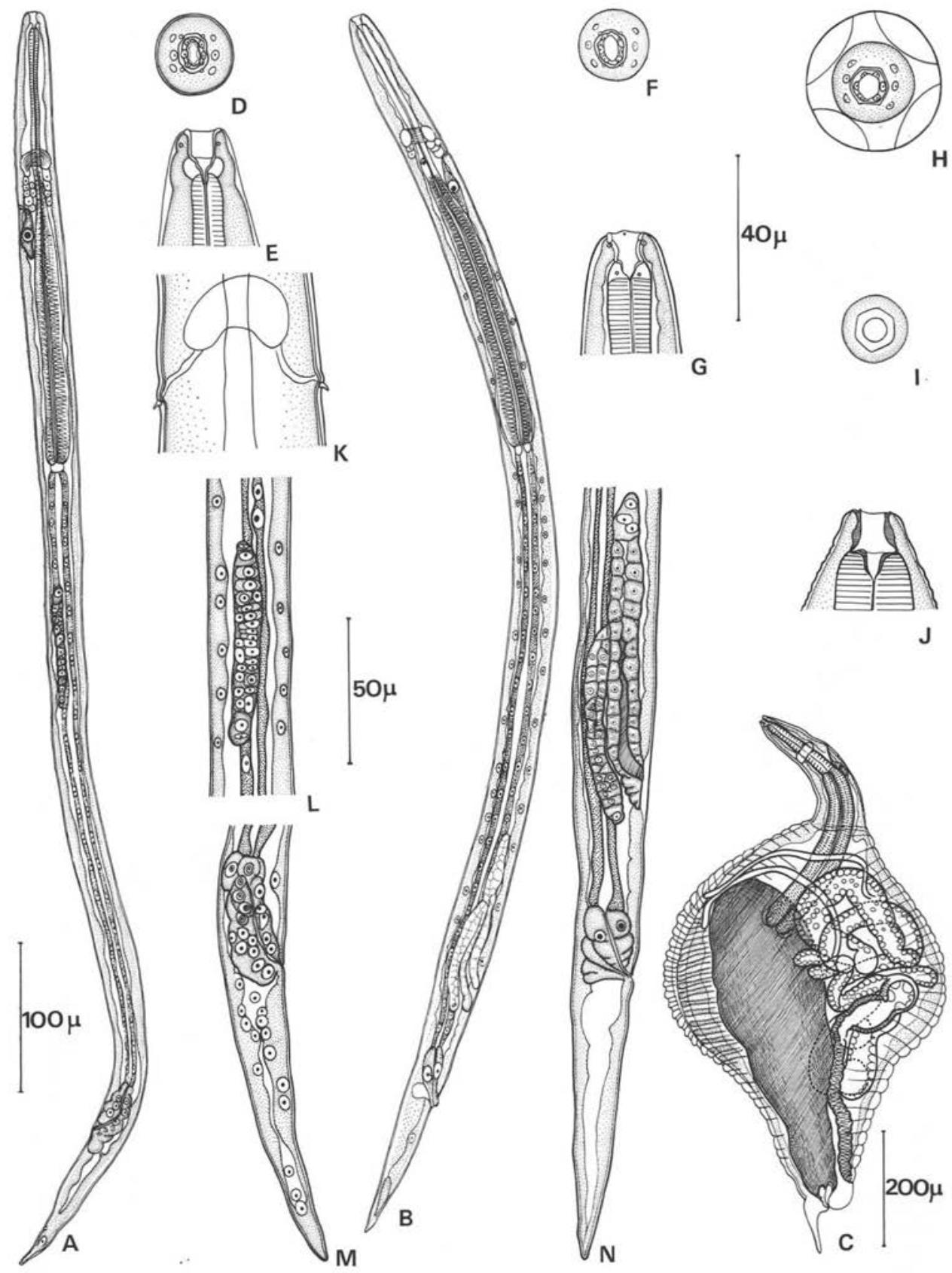


\section{Conclusion}

\section{Organogénèse.}

L'organogénèse de ce Spiruride est extrêmement rapide. La division de la cellule $\mathrm{R} 1$ et l'accroissement du nombre de cellules intestinales se réalisent en effet dès le début du premier stade larvaire. La division de ces initiales est plus lente dans le genre Spirura et la croissance des différentes ébauches larvaires est plus graduelle chez les Nématodes Rictulaires.

Le développement de ce Tetrameres est comparable à celui d'Acuaria anthuris, où la mise en place des ébauches larvaires s'effectue dès les trois premiers jours $(\boldsymbol{C} f$. Quentin et Poinar, 1973).

\section{Croissance et Morphogénèse.}

La taille des larves infestantes de $T$. cardinalis $(800 \mu)$ est identique à celle des larves de Tetrameres sp. Kartman, 1951 (740-891 $\mu)$. Elle est inférieure à celle des autres larves infestantes de Tetrameres dont les cycles sont connus. $\mathrm{La} \mathrm{L}_{3}$ mesure en effet $0,9-1,1 \mathrm{~mm}$ chez $T$. fissispina, $1,4 \mathrm{~mm}$ chez $T$. crami, 1,45 à $1,69 \mathrm{~mm}$ chez T. mohtedai, 1,8 à $1,9 \mathrm{~mm}$ chez $T$. americana.

Sur le plan de la morphogénèse, l'intérêt de $T$. cardinalis est de présenter des structures céphaliques particulièrement primitives; il n'existe en effet pas d'évolution labiale entre les structures céphaliques larvaires et celles des adultes mâles. Les structures céphaliques des adultes femelles sont légèrement atrophiées par rapport à celle des mâles.

Ces structures diffèrent donc profondément de celles d'espèces plus évoluées telles que $T$. crami, $T$. fissispina et $T$. americana, où le mâle présente deux pseudo-lèvres trilobées et deux interlabia. Chitwood et Wehr, 1934 ont d'ailleurs figuré la différenciation de ces structures de larve à l'adulte chez $T$. americana et ont mis en évidence l'évolution régressive de ces structures entre le mâle et la femelle plus spécialisée.

FiG. 7. - Tetrameres (Tetrameres) cardinalis n. sp. A et $\mathrm{B}$ : larves du quatrième stade $\delta$ et $q$, après 10 jours de développement chez le Cardinal; $\mathrm{C}:$ jeune $q$ récoltée après 14 jours de développement chez le Cardinal; $\mathrm{D}$ : tête en vue apicale du quatrième stade $\sigma^{2} ; \mathrm{E}$ : capsule buccale en vue dorsale de cette larve ; F : tête en vue apicale du quatrième stade $\$$; $\mathrm{G}$ : capsule buccale, en vue latérale, de cette larve; $\mathrm{H}$ : tête en vue apicale d'une jeune o âgée de 14 jours; I : coupe optique au niveau de la capsule buccale; J : capsule buccale de cette femelle, vue latérale; $K$ : détail des deirides en vue ventrale; L : ébauche génitale

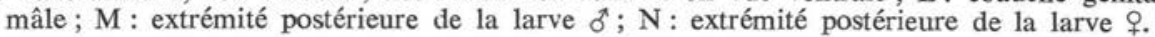

A, B : éch. $100 \mu ;$ C : éch. $200 \mu$; D, E, F, G, H, I, J, K : éch. $40 \mu ;$ L, M, N : éch. $50 \mu$. 


\section{Comportement larvaire chez l'hôte intermédiaire.}

Le comportement larvaire de $T$. cardinalis est comparable à celui de $T$. mohtedai, où les larves s'encapsulent dans le tissu adipeux d'Orthoptères. Ce comportement diffère de celui des espèces $T$. crami, $T$. fissispina et $T$. americana, dont les larves pénètrent dans les fibres musculaires de Copépodes ou d'Insectes.

L'adaptation des Tetrameres aux ússus de l'hôte intermédiaire est donc progressive ; la localisation dans le tissu adipeux représente, en effet, une étape avant la pénétration du parasite dans le tissu musculaire, comme le montre l'évolution constatée dans les localisations des Nématodes hétéroxènes chez l'hôte intermédiaire, selon la place qu'ils occupent dans l'échelle zoologique (Cf. Chabaud, 1954 ; Seureau, 1973) ; la migration du parasite chez l'hôte intermédiaire est d'autant plus importante que le Nématode occupe une place zoologique plus élevée.

Ainsi, dans le genre Tetrameres, une corrélation semble exister entre l'évolution des structures céphaliques et l'évolution du comportement larvaire, Les formes primitives ( $T$. cardinalis) se développent dans le tissu adipeux, alors que celles plus évoluées ( $T$. crami, $T$. fissispina et $T$. americana) s'encapsulent dans le tissu musculaire de l'hôte intermédiaire. Ces évolutions morphogénétiques et biologiques, et la diversité des espèces rencontrées témoignent de l'importance du genre Tetrameres, qui représente à lui seul une lignée parasitaire.

\section{Bibliographie}

Chabaud (A.-G.), 1954. - Sur le cycle évolutif des Spirurides et de Nématodes ayant une biologie comparable. Valeur systématique des caractères biologiques. Ann. Parasit. hum. comp., 29, 42-88, 206-249, 358-425.

Chitwood (B. G.) et WeHr (E. E.), 1934. - The value of cephalic structures as characters in Nematode classification, with special reference to the super-family Spiruroidea. Z. parasitenk., 7, 273-335.

Cram (E. B.), 1931, - Developmental stages of some Nematodes of the Spiruroidea parasitic in poultry and game birds. Techn. Bull., 227, 1-28.

Garkavi (B. L.), 1949. - Cycle évolutif de Tetrameres fissispina. Dokl. Akad. Nauk. SSSR, $64,1215-1218$.

Harwood (P. D.), 1933. - Some spiruroid Nematodes from Texas birds. Trans. Amer. Microscop. Soc., 52, 173-176.

Kartman (L.), 1951. - Notes on Tetrameres sp. (Nematoda, Spiruroidea) parasite in the English sparrow in Hawaii. Pacific Sci. Honolulu, 5, 252-255.

Lim (C. W.), 1975. - The fowl (Gallus domesticus) and a Lepidopteran (Setomorpha rutella) as experimental hosts for Tetrameres mohtedai (Nematoda). Parasit., 70, 143-148. 
OSHMARIN (P. G.), 1956. - Tetrameres (Spirurata, Tetrameridae) des Oiseaux domestiqueset sauvages de la région de Primorsk. Tr. Dal. Fil. A.N. SSSR, Ser. Zool., 3, 281-314. (en russe).

Quentin (J. C.) et Poinar (G. O.), 1973. - Comparative study of the larval development of some heteroxenous subulurid and spirurid Nematodes. Intern. J. Parasit., 3,. 809-827.

SANDGRound (J.H.), 1928. - A new Nematode parasite Tetrameres paucispina from a. South American bird, Amblyramphus holosericeus. J. parasitol., 14, 265-268.

ScHmidT (G. D.), 1962. - Tetrameres coloradensis sp. n., a Nematode parasite of thecommon snipe Capella Gallinago delicata. J. Parasit., 48, 250-851.

SEUREAU (C.), 1973. - Réactions cellulaires provoquées par les Nématodes Subulures et Spirurides chez Locusta migratoria (Orthoptère). Localisation et structure des. capsules. Z. Parasitenk., 41, 119-138.

Sundaram (R. K.), Radhakrishnan (C. V.), Sivasubramaniyam (M. S.), Padmanabhan (R.) et Peter (C. T.), 1963. - Studies on the life-cycle of Tetrameres mohtedai, Bhalerao et Rao, 1944. Ind. Veter. J., 40, 7-15.

Swales (W. E.), 1936. - Tetrameres crami Swales, 1933, a Nematode parasite of ducks. in Canada. Morphological and biological studies. Canad. J. Research, 14, 151-164.

Travassos (L.), 1917. - Tetrameridae brasileiras (2. Nota previa). Brasil. Med., 31, 65-66. 